\title{
Advanced undergraduate experiments in vacuum physics and mass spectrometry
}

\author{
I. Golecki \\ California Institute of Technology, Pasadena, California 91125
}

\author{
J. Rossel \\ Institut de Physique de l'Université, 2000 Neuchâtel, Switzerland \\ (Received 15 August 1979; accepted 29 November 1979)
}

\begin{abstract}
A comprehensive high-vacuum system has been set up and operated in an advanced undergraduate laboratory for students majoring in physics and microelectronics. The aim of the experiment is to provide the students with both practical experience and basic theoretical understanding of the production and measurement of low pressures. The students measure the pumping speed of a rotary forepump and of an oil diffusion pump, as a function of pressure, using procedures adopted by the AVS. A hot-cathode ionization gauge and a thermocouple gauge are calibrated against a McLeod (absolute) manometer for several gases. The compositions of ambient air, of an isotopic mixture of neon, and of the residual gases in an oil-diffusion-pumped system are determined with the aid of a mass spectrometer. The influence of a liquid-nitrogen-cooled surface is assessed. Helium leak detection is demonstrated, and the response and sensitivity of the mass spectrometer as a leak detector are evaluated.
\end{abstract}

PACS numbers: 01.50.Pa, 47.80. $+\mathrm{v}$, 07.30.Hd

\section{INTRODUCTION}

Knowledge of high-vacuum physics and familiarity with the related equipment is a necessity in the present day environment of research and technology. Thus an undergraduate university education in the physical sciences would not be considered complete without a course in vacuum physics, and first-hand experience in vacuum practice. In this paper we describe briefly a comprehensive high-vacuum system, which has been set up and operated in an undergraduate laboratory for third and fourth year students majoring in physics and microelectronics. During the course of the experiment, the students measure the pumping speed of a rotary forepump and of an oil diffusion pump. A hot-cathode ionization gauge and a thermocouple gauge are calibrated against a McLeod manometer. The compositions of various gas mixtures are determined with the aid of a mass spectrometer. Helium leak detection is demonstrated, and the response and sensitivity of the mass spectrometer as a leak detector are exaluated. The students thus gain both practical experience and basic theoretical understanding of the production and measurement of low pressures.

\section{SYSTEM DESCRIPTION}

The experimental system is shown schematically in Fig. 1. It is made of stainless steel or aluminum, with the exception of the McLeod gauge and $U$-tube monometer, which are made of glass. The measurement chamber is evacuated by means of a $100 \mathrm{l} / \mathrm{min}$, double-stage rotary pump (Edwards ED-100) and a 150 l/s oil diffusion pump (Leybold DI-150). An $\mathrm{Al}_{2} \mathrm{O}_{3}$ foreline trap and a water-cooled baffle on the diffusion pump are used to reduce backstreaming of oil from the forepump and the diffusion pump, respectively. The measurement chamber has a $400 \mathrm{~cm}^{2}$ finger, which can be filled with liquid nitrogen. A mass spectrometer head (Aerovac AST-1), a hot-cathode ionization gauge (Balzers IMR 110), and a thermocouple gauge (Varian NRC 531) are mounted on separate flanges. A fourth port equipped with a gate valve $G$, permits connection of additional equipment, such as a McLeod gauge (Leybold Model 1), or a calibrated conductance or leak for pumping speed measurements. Metal parts can be assembled quickly with the aid of standardized ISOKF small flanges, using neoprene O-rings. Metal-to-glass connections are made with epoxy cement or with pizein black wax. The whole system is mounted on a mobile stand.

\section{FOREPUMP SPEED}

The pumping speed of the rotary pump is measured as function of pressure using the constant volume method. ${ }^{1} \mathrm{~A}$ receptacle having a volume $V=10 \mathrm{l}$ is attached to the foreline for this purpose; it can also serve as a "buffer vacuum" for the diffusion pump. A mercury-filled U-tube manometer and a thermocouple gauge, which are connected to the recestacle, cover the pressure range $1-200$ and $10^{-3}-2$ Torr, respectively. Calibration curves for the thermocouple gauge for several gases are provided by the manufacturer, and measured in the following. The receptacle is filled with nitrogen or dry air and the time $t$, required to pump it down to pressure $p$, from reference pressure $p_{i}$, is measured. From the plot of $p$ vs $t$ the effective pumping speed $S$ is calculated using Eq. (1):

$$
S(p)=-(V / p)(d p / d t) .
$$




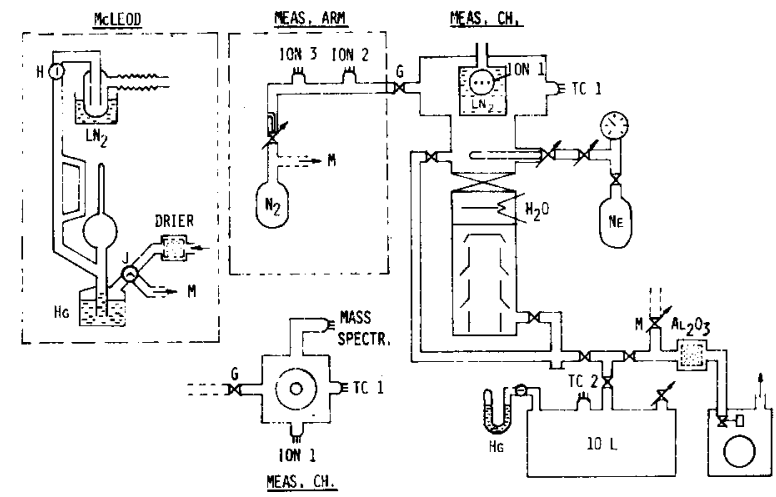

FIG. 1. Schematic diagram of the educational high-vacuum apparatus (not to scale). See text for details.

For $p$ much larger than the ultimate pressure, $S$ is independent of pressure, and a plot of $\ln p$ vs $t$ yields a straight line with slope equal to $-(S / V)$. The results are $S \cong 70 \mathrm{l} / \mathrm{min}$ for $p \geqslant 4 \times 10^{-2}$ Torr, with $S$ decreasing at lower pressures (e.g. $S=35 \mathrm{l} / \mathrm{min}$ at $p=6 \times 10^{-3}$ Torr), due to the finite compression ratio and the inevitable leakage through the internal seals of the pump. The effective pumping speed is lower than the nominal speed, due to flow resistance of the tubing between pump inlet and receptacle. At the lower end of the pressure range, the contribution of the vapor pressure of the forepump oil reduces the accuracy of measurement.

\section{DIFFUSION PUMP SPEED}

The pumping speed of the diffusion pump for nitrogen or dry air is measured as function of pressure by using (a) a calibrated conductance ${ }^{2,3}$ and (b) a calibrated leak. ${ }^{3}$ For this part of the experiment, the measurement arm is attached to the chamber through gate valve $G$. In method (a), gas is admitted into the chamber through the arm via a needle valve at constant throughput. The pressures indicated by the identical ionization gauges 1,2 , and 3 are noted, and the effective pumping speed calculated from Eq. (2):

$$
S\left(p_{1}\right)=C_{32}\left(p_{3}-p_{2}\right) / p_{1} .
$$

$\mathrm{C}_{32}(\mathrm{l} / \mathrm{s})$ is the conductance of the tube between points 3 and 2 , given by the Knudsen empirical formula ${ }^{4}$

$$
C_{32}=C_{L}+C_{M}(1+256 d \bar{p}) /(1+316 d \bar{p})
$$

where $d(\mathrm{~cm})$ is the internal diameter of the tube, $\bar{p}$ (Torr) is the average pressure between points 3 and $2, C_{L}$ and $C_{M}$ are the tube conductances in the hydrodynamic (laminar) and molecular regimes, respectively:

$$
\begin{aligned}
& C_{L}=182\left(d^{4} / l\right) \bar{p} \\
& C_{M}=12.1\left(d^{3} / l\right)
\end{aligned}
$$

where $l(\mathrm{~cm})$ is the tube length between points 3 and 2 . In our setup $l(=64 \mathrm{~cm}) \gg d(=2 \mathrm{~cm})$, and therefore the long tube approximation used in Eqs. (4) and (5) is valid to within a few percent. Equations (3)-(5) are for dry air at $20^{\circ} \mathrm{C}$.

The measurements yield an approximately constant speed of $55 \mathrm{l} / \mathrm{s}$ between $10^{-5}$ and $10^{-3}$ Torr, decreasing rapidly at higher pressures (e.g. $27 \mathrm{l} / \mathrm{s}$ at $10^{-2}$ Torr and $10 \mathrm{l} / \mathrm{s}$ at $5 \times 10^{-2}$
Torr). The rather low value of the speed at low pressures, as compared to the nominal value, is due to (1) the conductances of the baffle $(170 \mathrm{l} / \mathrm{s})$ and the short, wide tubing between the baffle and the chamber $(200 \mathrm{l} / \mathrm{s})$, and possibly also to $(2)$ the incorrectly low value of $C_{32}$ obtained from the Knudsen formula (Eq. 3), as pointed out by Denison. ${ }^{5}$ An insight into the flow in the transition (slip) regime is obtained by noting the slight minimum in the calculated $C_{32}(\bar{p})$ curve at $\bar{p} \cong 2$ $\times 10^{-3}$ Torr. The pressure range $p_{1}$ usefully covered is $10^{-5}-10^{-1}$ Torr. The range of the ionization gauges is $10^{-6}-1$ Torr, but the accuracy below $10^{-5}$ Torr is limited by the ultimate pressures in the system, due to outgassing, whereas at the high pressure end the pumping speed is too unstable. The advantage of method (a) is that in principle absolute values of the pressure are not needed [Eq. (2)].

In method (b), either nitrogen or dry air is admitted into the measurement arm through a glass capillary, having a known throughput $Q(\operatorname{Torr} \mathrm{l} / \mathrm{s})$. The pumping speed at point 1 is then given by

$$
S\left(p_{1}\right)=Q / p_{1} .
$$

A series of capillaries with $Q$ values from $7 \times 10^{-4}$ to $2 \times 10^{-1}$ Torr $1 / \mathrm{s}$ were prepared; they were calibrated using an "inverted buret", constant pressure method. ${ }^{6}$ The results of measurements using the two methods, (a) and (b), agree to within $15 \%$.

\section{CALIBRATION OF HOT-CATHODE IONIZATION GAUGE AND THERMOCOUPLE GAUGE}

For this part of the experiment, the McLeod gauge, ${ }^{7}$ containing $6 \mathrm{~kg}$ of mercury, is connected to the measurement chamber via gate valve $G$. A glass, liquid-nitrogen-cooled trap prevents mecury from the McLeod from contaminating the rest of the apparatus, and also reduces the water-vapor pressure in the gauge. For accurate measurements, stopcock $\mathrm{H}$ is closed before raising the mercury in the McLeod. This reduces the error due to "diffusion pumping" by the cold trap. ${ }^{8,9}$ The finger in the measurement chamber is also cooled with liquid nitrogen to lower the background pressure to the low $10^{-7}$ Torr range. The useful range of the McLeod is $10^{-5}-10^{-1}$ Torr, with a probable error of $3 \%$ or $2 \times 10^{-5}$ Torr (whichever is larger). The gauge has a calibrated linear scale. The indications of the ionization gauge 1 , thermocouple gauge 1 , and the McLeod gauge are compared over the overlapping pressure ranges for nitrogen and neon or helium. Beyond the immediate aim of calibrating the two relative pressure gauges, and finding the quadratic constant of the McLeod $\left(4 \times 10^{-6}\right.$ $\mathrm{mm}^{-1}$ ), the experiment points to the caution which should be exercised when evaluating pressure readings of relative gauges in ambients of unknown composition. The readings of the Balzers IMR 110 ionization gauge for nitrogen are found to be within $10 \%$ of the McLeod values, while the sensitivity with respect to nitrogen is 0.25 for neon and 0.17 for helium, in agreement with the manufacturer's calibration. The ion current vs. pressure characteristic is linear over the pressure range studied. The voltage output of the thermocouple gauge is non-linear with pressure for all gases considered, and agrees qualitatively with the manufacturer's curves. 


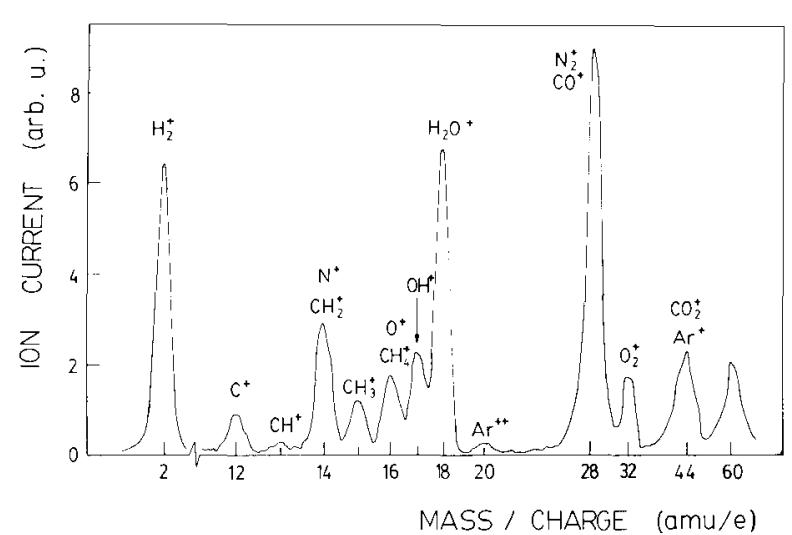

FIG. 2. Mass spectrum of the residual gases in the measurement chamber with the $400 \mathrm{~cm}^{2}$ finger cooled with liquid nitrogen. The total pressure, measured by a hot cathode ionization gauge, is $10^{-6}$ Torr. The presence of light and heavy (mass/charge $=60$ ) hydrocarbon radicals is probably due to a contamination of the mass spectrometer head by oil vapors from the diffusion pump.

\section{MASS SPECTROMETRY}

This last part of the experiment is designed to acquaint the students with several simple uses of a mass spectrometer. The spectrometer is provided with two fixed magnets, covering together the mass range 2-70 amu. The unit mass resolution, taken as the full peak width at quarter height, is 20 at $M=20$ amu. The electron accelerating voltage is fixed at $65 \mathrm{~V}$, while the ion accelerating voltage $V_{i}$, can be varied between 65 and $400 \mathrm{~V}$, either manually or automatically. In the latter mode, a scanning time of $2 \mathrm{~min}$ is used, and spectra are plotted on a stripchart recorder. The sensitivity of the instrument for nitrogen is $10^{-10}$ Torr.

The experiment consists of four parts, (a) to (d). In part (a), the composition of an isotopic mixture of neon is measured $(M=20,21,22$ with natural abundances $90.5 \%, 0.26 \%, 9.2 \%)$, and the limit of resolution of the spectrometer is evaluated. In part (b), the mass spectrum of ambient air is recorded, and the main constituents are located. Minor constituents are then identified by using the relation $\mathrm{M} \propto 1 / V_{i}$. The composition of air is calculated by using the relative sensitivities of the spectrometer, supplied by the manufacturer. The concept of cracking pattern ${ }^{10,11}$ is introduced (e.g. for $\mathrm{H}_{2} \mathrm{O}$ ). In part (c) the composition of the residual gases in the measurement chamber is measured. It is shown that the main constituent in a room-temperature, oil-diffusion-pumped chamber is water vapor. The effect of a liquid-nitrogen-cooled surface in reducing the partial pressures of condensable gases is demonstrated [e.g. $p\left(\mathrm{H}_{2} \mathrm{O}\right)$ is reduced by a factor of 50 ]. Cracking patterns are used to demonstrate the possible exis- tence of high-mass hydrocarbon ions which cannot be detected directly. A typical spectrum of the residual gases in the system when using the liquid-nitrogen-cooled finger is shown in Fig. 2.

In part (d), the mass spectrometer is tuned to the mass of helium, and its response as a leak detector is measured with a simulated leak. The response is of the form

$$
p(\mathrm{He})=p_{0}(\mathrm{He}) \exp \left(-t / T_{1}\right)\left[1-\exp \left(-t / T_{2}\right)\right]
$$

where $T_{1}$ and $T_{2}$ depend on the magnitude of the leak and the pumping time constant $(V / S)$. Systematic leak detection is demonstrated, and the sensitivity of the mass spectrometer as leak detector is calculated.

\section{SUMMARY}

An educational, high-vacuum apparatus is described, which incorporates most of the important elements found in the research or technological environment. After having completed the experiments and evaluated the data, the students have been exposed to the basic theoretical concepts and practical techniques underlying vacuum technology. The students are further encouraged to explore beyond the experiment itself in a series of questions of the short essay type. These include other pumping methods (such as turbomolecular, ion, or cryogenic) and further experiments which can be performed with the mass spectrometer.

\section{ACKNOWLEDGMENT}

The authors wish to acknowledge the expert technical assistance of J. S. Margot, and the financial support of the Canton of Neuchâtel, Department of Education.

Work performed at Institut de Physique de l'Université, 2000 Neuchâtel, Switzerland.

'J. Yarwood, High Vacuum Technique (Chapman and Hall, London, 1967) 4th ed., pp. 158-159.

${ }^{2}$ S. L. Rutherford, Vacuum 16, 643 (1966).

${ }^{3}$ A VS Tentative Standard 4.1 (1963) J. Vac. Sci. Technol. 8, 664 (1971).

${ }^{4}$ J. Delafosse and G. Mongodin, Les Calculs de la Technique du Vide (Société Française des Ingénieurs et Techniciens du Vide, 1961) p. 35.

${ }^{5}$ D. R. Denison, J. Vac. Sci. Technol. 12,548 (1975)

${ }^{6}$ A. Roth, Vacuum Technology (North Holland: Amsterdam, 1976) p. 268

${ }^{7}$ S. Dushman, Scientific Foundations of Vacuum Technique, edited by $\mathbf{J}$. M. Lafferty (Wiley, New York, 1962) 2nd ed., p. 226.

${ }^{8}$ See pp. $96-98$ in Ref. 1.

${ }^{9} \mathrm{C}$. Meinke and G. Reich, Vakuum-Technik 12,79 (1963).

${ }^{10}$ See e.g. R. D. Craig and E. H. Harden, Vacuum 16, 67 (1966).

${ }^{11} \mathrm{H}$. H. Willard, L. L. Merritt Jr., and J. A. Dean, Instrumental Methods of Analysis (Van Nostrand, Princeton, NJ, 1965) 4th ed., Chap. 16. 